\title{
Boundary conditions for two-dimensional integrable chains
}

\author{
Burak Gürel ${ }^{\mathrm{a}, 1}$, Ismagil Habibullin ${ }^{\mathrm{b}, 2}$ \\ a Department of Mathematics, Faculty of Science, Bilkent University, 06533 Ankara, Turkey \\ ${ }^{\mathrm{b}}$ Ufa Institute of Mathematics, Russian Academy of Sciences, Chernyshevsky Street, 112, 450000 Ufa, Russian Federation
}

Received 3 February 1997; revised manuscript received 3 June 1997; accepted for publication 6 June 1997

Communicated by A.P. Fordy

\begin{abstract}
The symmetry method of studying boundary value problems is generalized to the multi-dimensional case. In passing from $1+1$ dimensions to $2+1$ dimensions the main obstacle is the existence of nonlocal variables. To overcome this obstacle we have derived additional constraints that link the nonlocal variables of different levels. As an illustration, the application of the method to the two-dimensional Toda lattice is considered. (C) 1997 Elsevier Science B.V.
\end{abstract}

\section{Introduction}

At present classes of boundary conditions are known for integrable nonlinear partial differential equations and lattice equations with two independent variables, in both classical and quantum versions, compatible with the integrability property. In the last decade the subject has become rather popular; various approaches were worked out and applied successfully (see Refs. [1,2]). An effective method to investigate boundary value problems for integrable $1+1$ dimensional nonlinear equations was proposed and developed in Refs. [3,4] based on the symmetry approach. The symmetry test established in Refs. [3,4] allows one, in principle, to describe the complete set of boundary conditions for the given equation, compatible with its integrability property. For instance, recently the boundary value problem for the Korteweg-de Vries equation has successfully

\footnotetext{
${ }^{1}$ E-mail: gurel@fen.bilkent.edu.tr.

${ }^{2}$ E-mail: ihabib@imat.rb.ru.
}

been studied within the framework of the symmetry approach $[5,6]$.

However, the initial boundary value problem for integrable systems (i.e. systems admitting a symmetry algebra having infinitely many elements [7]) with more than two independent variables were not studied much. In the present paper we have undertaken an attempt to generalize the symmetry test to the multidimensional case. As a touchstone we take a very well studied two-dimensional integrable phenomenon, the 2D Toda lattice equation,

$q_{x y}(n)=\mathrm{e}^{q(n+1)-q(n)}-\mathrm{e}^{q(n)-q(n-1)}$.

Various alternative forms of the Toda lattice and interrelations between them are discussed in Ref. [8] Besides the inherent interest in integrable systems, the Toda system has a wide number of applications in many fields from quantum field theory to differential geometry. Here and below the subscripts $x, y, t$ denote partial derivatives with respect to these variables.

By a boundary condition we mean a constraint of the following form, 


$$
\begin{gathered}
F\left(q(m), q(m+1), \ldots q(k), q_{x}(m), \ldots\right. \\
\left.q_{x}(k), q_{y}(m), \ldots q_{y}(k), \ldots\right)=0,
\end{gathered}
$$

depending on a finite number of the dynamical variables $q(n)$ and a finite number of their $x$ - and $y$ derivatives, which splits the infinite lattice (1) into two semi-infinite ones. Notice that here numbers $m$ and $k$ with $m \leqslant k$ are fixed (but for any $m$ and $k$ satisfying $m \leqslant k$ this can be done). These two semiinfinite lattices are

$$
\begin{aligned}
& q_{x y}(n)=\mathrm{e}^{q(n+1)-q(n)}-\mathrm{e}^{q(n)-q(n-1)}, \quad n \leqslant k-1, \\
& q(k)=F_{1}\left(q(m), q(m+1), \ldots, q_{x}(m), \ldots,\right. \\
& \left.\quad q_{y}(m), \ldots\right)
\end{aligned}
$$

and

$$
\begin{aligned}
& q_{x y}(n)=\mathrm{e}^{q(n+1)-q(n)}-\mathrm{e}^{q(n)-q(n-1)}, \quad n \leqslant m-1, \\
& q(m)=F_{2}(q(m+1), q(m+2), \ldots, \\
& \left.\quad q_{x}(m+1), \ldots, q_{y}(m+1), \ldots\right),
\end{aligned}
$$

where $q(k)$ and $q(m)$ are expressed through Eq. (2).

Notice that an equation of the form $q_{t}(n)=a$, where the right hand side $a$ depends on a finite number of variables $q(n)$, and their derivatives, and on socalled nonlocal variables, is a symmetry of Eq. (1) if the commutativity condition $\left(q_{t}(n)\right)_{x y}=\left(q_{x y}(n)\right)_{t}$ is valid. Our further considerations are based on the following:

Definition. The boundary condition (2) is considered to be compatible with the symmetry if the commutativity condition is still valid subject to the constraint (2). Or, in other words the semi-infinite lattice (3) and the following semi-infinite lattice

$$
\begin{aligned}
& q_{t}(n)=a, \quad n \leqslant k-1, \\
& q(k)=F_{1}\left(q(m), q(m+1), \ldots, q_{x}(m), \ldots,\right. \\
& \left.\quad q_{y}(m), \ldots\right)
\end{aligned}
$$

commute. A similar commutation condition may be given also for the semi-infinite chain (4) but this condition is automatically satisfied after imposing the condition for Eq. (3).

In this paper we consider two classes of boundary conditions, consistent with the test symmetry ( see
Eq. (6) below). A complete set of such conditions of the form $q(1)=g\left(q(0), q_{x}(0), q_{y}(0), q(-1)\right)$ is given at the end of Section 3. They all correspond to the integrable finite Toda lattices, connected with the Cartan matrices of simple Lie algebras of finite growth [9]. Boundary conditions of the other kind $q_{y}(1)=g\left(q(1), q(0), q_{x}(0)\right)$ are studied in Section 4 . In this case one has the only boundary condition (34) compatible with Eq. (6), see also Ref. [10]. It does not belong to the class of finite Toda chains mentioned above.

\section{Boundary conditions consistent with symmetries}

In two dimensions the main obstacle is the existence of nonlocal variables on which higher symmetries depend. For instance, the following two equations,

$q_{t_{1}}(n)=b_{1}(n)+b_{1}(n-1)+q_{x}(n)^{2}$

and

$$
\begin{aligned}
& q_{t}(n)=b_{2}(n-2)+b_{2}(n-1)+b_{2}(n) \\
& \quad+b_{1}(n)\left[2 q_{x}(n)+q_{x}(n+1)\right] \\
& \quad+b_{1}(n-1)\left[2 q_{x}(n)+q_{x}(n-1)\right]+q_{x}(n)^{3}
\end{aligned}
$$

are two symmetries of the Toda lattice (1). They depend on two nonlocalities $b_{1}(n)$ and $b_{2}(n)$, which are introduced as solutions to the equations [8]

$$
\begin{aligned}
& q_{x x}(n)=b_{1}(n)-b_{1}(n-1), \\
& b_{1, y}(n)=c(n)\left[q_{x}(n+1)-q_{x}(n)\right], \\
& b_{1, x}(n)=b_{1}(n)\left[q_{x}(n+1)-q_{x}(n)\right]+b_{2}(n) \\
& \quad-b_{2}(n-1), \\
& b_{2, y}(n)=c(n) b_{1}(n+1)-c(n+1) b_{1}(n),
\end{aligned}
$$

where $c(n)$ is described by the equation $c(n)=$ $\mathrm{e}^{q(n+1)-q(n)}$. Other nonlocal variables $b_{j}, j>1$ satisfy the similar equations,

$$
\begin{aligned}
& b_{j, x}(n)=b_{j}(n)\left[q_{x}(n+j)-q_{x}(n)\right]+b_{j+1}(n) \\
& \quad-b_{j+1}(n-1), \\
& b_{j+1, y}(n)=c(n) b_{j}(n+1)-c(n+j) b_{j}(n) .
\end{aligned}
$$


Let us pass from the standard set of local dynamical variables

$q(n), q_{x}(n), q_{x x}(n), \ldots, q_{y}(n), q_{y y}(n), \ldots$

for all $n=0, \pm 1, \pm 2, \ldots$

to the set consisting of variables $u, v$ and all their $x$ and $y$-derivatives by

$u=\mathrm{e}^{q(1)}, \quad v=\mathrm{e}^{-q(0)}$.

For example, $q(-1)$ may be eliminated by means of the Toda equation itself by setting

$\mathrm{e}^{-q(-1)}=\mathrm{e}^{q(1)-2 q(0)}-q_{x y}(0) \mathrm{e}^{-q(0)}$,

and so on. In terms of thesc new variables the symmetries (5) and (6) become (see also Ref. [11])

$u_{t_{1}}=u_{x x}+2 r u, \quad v_{t_{1}}=-v_{x x}-2 r v$,

$u_{t}=u_{x x x}+3 r u_{\lambda}+3 s u$,

$v_{t}=v_{x x x}+3 r v_{x}-3 s v+3 r_{x} v$,

where nonlocalities $r=b_{1}(0)$ and $s=b_{2}(0)+$ $r(\log u)_{x}$ obviously satisfy the equations

$r_{y}=(u v)_{x}$,

$s_{y}=\left(u_{x} v\right)_{x}$.

The boundary condition (2) takes the form

$\tilde{F}\left(u, v, u_{x}, v_{x}, u_{y}, v_{y}, \ldots\right)=0$.

A very useful consequence of the change of variables is the following statement:

Proposition. The boundary condition (2) is compatible with the symmetry (6) (or (5)) if and only if the constraint (16) is consistent with the system (13) (or (12)).

Below in the next two sections we will consider two special cases of the constraint (16)

$u=f\left(v, v_{x}, u_{y}, v_{x y}\right)$,

$u_{y}=f\left(u, v, v_{x}\right)$.

In either case we will use Eq. (13) as the test symmetry because the other one (12) does not admit any constraint of the above forms for it is skew-symmetric in the highest order derivatives (cf. Ref. [12]).

\section{Differential constraints of the form}

$u=f\left(v, v_{x}, v_{y}, v_{x y}\right)$

Suppose that the constraint (17) is consistent with the system (13). Let us differentiate it with respect to $t$ by means of the system (13) and then replace the variables $u, u_{x}, u_{y}, \ldots$ in the resulting equation through variables

$s, s_{x}, r, r_{x}, r_{x x}, v, v_{x}, v_{y}, v_{x x}, \ldots$

This will either produce one more differential constraint of the form

$G\left(s, s_{x}, r, r_{x}, r_{x x}, v, v_{x}, v_{y}, v_{x x}, \ldots\right)=0$,

containing a finite number of variables, or is satisfied identically. In the former case, Eq. (19) can essentially be simplified. Actually the following statement takes place:

Lemma. Unless Eq. (19) is valid identically it is of one of the forms:

$s=H\left(r, r_{x}, v, v_{x}, v_{x x}, v_{x x x}\right)$,

$r=H\left(v, v_{x}, v_{x x}\right)$.

Proof. If $\partial G / \partial s_{x}=0$ then Eq. (19) can be written as (if $\partial G / \partial s=0$ then Eq. (24) holds)

$s=H_{1}\left(r, r_{x}, r_{x x}, v, v_{x}, v_{y}, \ldots\right)$

and a comparison with Eq. (15) immediately gives Eq. (20). If $\partial G / \partial s_{x} \neq 0$, then Eq. (19) is equivalent to an equation of the form

$s_{x}=H_{2}\left(s, r, r_{x}, r_{x x}, v, v_{x}, v_{y}, \ldots\right)$.

The compatibility condition of Eq. (15) with (23) gives either an equation of the form (22) or an equation like

$r_{x x}=H_{3}\left(r, r_{x}, v, v_{x}, v_{y}, \ldots\right)$.

Finally, a comparison of Eqs. (24) and (14) leads to Eq. (21).

Thus according to the Lemma the problem of classifying the constraint (17) splits into the following subcases: 
(c1) to find the function $u=f\left(u, v_{x}, v_{y}, v_{x y}\right)$, for which the equation

$D_{t} f-D_{x}^{3} f+3 r D_{x} f+3 s f$,

where $D_{t}$ and $D_{x}$ are total derivative operators with respect to $t$ and $x$, holds identically for all values of the variables

$s, s_{x}, r, r_{x}, r_{x x}, v, v_{x}, v_{y}, v_{x x}, \ldots$

(c2) to find pairs of functions

$u=f\left(v, v_{x}, v_{y}, v_{x y}\right)$,

$s=H\left(r, r_{x}, v, v_{x}, v_{x x}, v_{x x x}\right)$,

such that the equations

$D_{t} f=D_{x}^{3} f+3 r D_{x} f+3 s f$,

$D_{y} H=D_{x}\left(v D_{x} f\right)$,

where $D_{y}$ is the total derivative operator with respect to $y$, are valid identically for all values of variables

$r, r_{x}, r_{x x}, v, v_{x}, v_{y}, v_{x x}, \ldots$

modulo Eqs. (25) and (26) and their $t$ - and $y$ differential consequences, respectively.

(c3) to find pairs of functions

$u=f\left(v, v_{x}, v_{y}, v_{x y}\right)$,

$r=H\left(v, v_{x}, v_{x x}\right)$,

such that Eq. (27) and the equation $D_{y} H=D_{x}(f v)$ are valid identically for all values of variables

$s, s_{x}, v, v_{x}, v_{y}, v_{x x}, \ldots$

modulo Eqs. (29) and (30), and their $t$ - and $y$ differential consequences, respectively.

Direct computations lead to the following cases: the only choice in the case of $(c 1)$ is

(i) $u=0, s_{y}=0$;

in the second case one has three solutions:

(ii) $u=a, \quad s=0$;

(iii) $u=a v, s=\frac{1}{2} r_{x}$; (iv) $u=\frac{v_{x y}}{\left(a-v^{2}\right)}+\frac{v v_{x} v_{y}}{\left(a-v^{2}\right)^{2}}$,

$$
s=r_{x}-\frac{v_{x} v_{x x}}{a-v^{2}}-\frac{v v_{x}^{3}}{\left(a-v^{2}\right)^{2}} \text {. }
$$

Here $a$ is an arbitrary constant. Lastly the third case (c3) gives the following solution:

(v) $u=-\frac{v_{x y}}{v^{2}}+\frac{v_{x} v_{y}}{v^{3}}, \quad r=-\frac{v_{x x}}{v}+\frac{v_{x}^{2}}{v^{2}}+b$,

where $b$ is an arbitrary constant.

Turning back to the original variables, the differential constraints (i) $-(v)$ correspond to the boundary conditions for the Toda system (1). Below we give the boundary conditions together with the equations for the associated nonlocalities:

(1) $\mathrm{e}^{q(1)}=0, \quad b_{2, y}(0)=0$

(2) $q(1)=$ const, $\quad b_{2}(0)=0$;

(3) $q(1)=-q(0)+$ const,

$$
b_{2}(0)=\frac{1}{2} b_{1, x}(0)+b_{1}(0) q_{x}(0)
$$

$$
\begin{aligned}
a \mathrm{e}^{q(1)} & =\mathrm{e}^{-q(-1)}+\frac{a q_{x}(0) q_{y}(0)}{a \mathrm{e}^{q(0)}-\mathrm{e}^{-q(0)}}, \\
b_{2}(0) & =b_{1, x}(0)-b_{1}(0) q_{x}(1) \\
+ & \frac{a q_{x}(0)^{3}}{\left(a \mathrm{e}^{q(0)}-\mathrm{e}^{-q(0)}\right)^{2}} \\
- & \frac{q_{x}(0) q_{x x}(0) \mathrm{e}^{-q(0)}}{a \mathrm{e}^{q(0)}-\mathrm{e}^{-q(0)}}
\end{aligned}
$$

$$
\mathrm{e}^{-q(-1)}=0, \quad b_{1}(0)=q_{x x}(0)+\text { const, }
$$

respectively. All boundary conditions (1) $-(5)$ are well known. They are nothing but the closure conditions imposed at two different points $n=n_{1}$ and $n=n_{2}$, which reduce the infinite Toda lattice to the socalled generalized (finite) Toda lattices. These finite Toda lattices are integrable and described by Cartan matrices of simple Lie algebras of finite growth [9].

Remark. In order to examine the compatibility condition with the next symmetries it is necessary to consider next nonlocalities. 


\section{Differential constraints of the form} $u_{y}=f\left(u, v, v_{x}\right)$

In this section we will consider differential constraints (18). Supposing that Eq. (18) is compatible with $t$-evolution and differentiating it with respect to $t$ one has again two choices: either the resulting equation holds identically or it gives an additional constraint

$$
\begin{aligned}
& G\left(s, s_{x}, r, r_{x}, r_{x x}, u, u_{x}, u_{x x}, \ldots v, v_{x}, v_{y}, v_{x x}, \ldots\right) \\
& \quad=0 .
\end{aligned}
$$

If it does not hold identically then it can be written in one of the forms (cf. Lemma in the previous section):

$s=H\left(r, r_{x}, u, u_{x}\right)$,

or

$$
r=H\left(u, u_{x}\right) \text {. }
$$

Tediously long but direct calculations show that the case (33) gives nothing and the case (32) contains exactly one differential constraint

$u_{y}=a v_{x}, \quad s=r_{x}-\frac{u u_{x}}{a}$.

In terms of the original lattice variables this constraint gives the following boundary condition,

$q_{y}(1)=-a \mathrm{e}^{-q(1)-q(0)} q_{x}(0)$,

which was found earlier in Ref. [10] by using the Bäcklund transform but it is still less studied. For the nonlocalities one has the following equation,

$$
b_{2}(0)=b_{1, x}(0)-b_{1}(0) q_{x}(1)-\frac{\mathrm{e}^{2 q(1)} q_{x}(1)}{a} .
$$

\section{Acknowledgement}

The authors are grateful to Professor M. Gürses for his interest in this work and useful discussions, and to Dr. O. Tekman for his help with MAPLE. This work has partially been supported by the Turkish Scientific and Technical Research Council (TUBITAK). One of the authors, I.H., thanks the Bilkent University for financial support and warm hospitality.

\section{References}

[1] E.K. Sklyanin, Func. Anal. Prilozh. 21 (1987) 86.

[2] S. Ghoshal and A.B. Zamolodchikov, Boundary state and boundary $\mathrm{S}$ matrix in two-dimensional integrable field theory (1993), RU-93-20, hep-th/9306002.

[3] I.T. Habibullin, Phys. Lett. A 178 (1993) 369.

[4] B. Gürel, M. Gürses and I. Habibullin, J. Math. Phys. 36 (1995) 6809; Phys. Lett. A 190 (1994) 231.

[5] V.E. Adler, B. Gürel, M. Gürses and I. Habibullin. J. Phys. A: Math. Gen. 30 (1997) 3505.

[6] V.E. Adler, I.T. Habibullin and A.B. Shabat, Theor. Math. Phys. 110 (1997) N1.

[7] A.V. Mikhailov, A.B. Shabat and R.I. Yamilov, Comm. Math. Phys. 115 (1988) 1.

[8] A.B. Shabat, Phys. Lett. A 200 (1995) 121.

[9] A.N. Leznov and M.V. Saveliev, Group methods of solving nonlinear dynamical systems (Nauka, Moscow, 1985).

[10] I.T. Habibullin, Phys. Lett. A 207 (1995) 263.

[11] A.N. Leznov, A.B. Shabat and R.I. Yamilov, Phys. Lett. A 174 (1993) 397.

[12] V.E. Adler and I.T. Habibullin, J. Phys. A: Math. Gen. 28 (1995) 6717. 